\title{
Conectados por redes sociales: Introducción al Análisis de redes sociales y casos prácticos
}

\author{
Belén Peyró Outeiriño ${ }^{1}$ \\ Departamento de Trabajo Social Facultad de Derecho, UNED
}

\section{Reseña}

Del-Fresno, M., Marqués, P. \& Sánchez, D (2014). Conectados por redes sociales: Introducción al Análisis de redes sociales y casos prácticos. Barcelona: Editorial UOC. 352 pág.

Como mecanismo de adaptación y supervivencia formamos parte de diferentes grupos, desde la familia a los amigos, en todos los ámbitos, académicos, profesionales, culturales, etc. De este modo, los seres humanos estamos inmersos de manera permanente en una red de relaciones, y establecemos conexiones con nuestros iguales pues como seres sociales necesitamos relacionarnos, tomar contacto con el otro. Formamos parte de redes, y en ellas definimos y son definidos nuestros roles, nuestras relaciones, y en base a estas, obtenemos y transmitimos información relevante para diversos ámbitos de nuestra vida.

Ya no se habla del fenómeno de la globalización, de tecnología, de internet, de web 2.0 como si de algo novedoso se te tratara, son elementos y conceptos que forman parte intrínseca de nuestras vidas, los hemos ido integrando en nuestras relaciones, en nuestra forma de comprender el mundo, de comunicarnos, en nuestro vocabulario común y en nuestra forma de actuar en variedad de contextos, sin llegar a comprender en toda su medida la amplitud y complejidad del fenómeno, de lo que Castell denomino la nueva forma de comunicación interactiva, la autocomunicación de masas (Castells, 2009). La nueva forma de comunicarnos y relacionarnos a nivel global, de forma asincrónica o sincrónica, ha conformado un nuevo tipo de sociedad, nos encontramos ante una nueva estructura social dominante: la sociedad red; una nueva economía: la economía informacional/global, y una nueva cultura: la cultura de la virtualidad real (Castells, 1998). En la nueva sociedad de red, la sociedad actual,

\footnotetext{
${ }^{1}$ Enviar correspondencia a: peyrobelen@gmail.com
}

DOI: http://dx.doi.org/10.5565/rev/redes.548 
construimos, deconstruimos, creamos, nos incorporamos, salimos, pertenecemos y dejamos de pertenecer a redes sociales. El espacio virtual nos permite movernos por el ciberespacio, un espacio sin límites tangibles y sin fronteras, en el que el acceso a la información y la vinculación con otros en red, se ha vuelto fundamental para lograr nuestro desarrollo como ciudadanos en un mundo globalizado y tecnológico.

El libro que vamos a reseñar, es un avance significativo en la difusión del conocimiento de las Redes Sociales, su conjunto de actores y sus vínculos relacionales, y del Análisis de Redes Sociales (ARS) como un método para medir, representar, analizar y entender los comportamientos sociales, los patrones de interacción social de los actores dentro de un determinado contexto.

¿Qué información se puede estudiar mediante el ARS?, ¿En que ámbitos o áreas puede aplicarse? ¿Cómo se usa y con qué fines? ¿Cuál es la potencialidad del método? En los once capítulos que estructuran el libro, veintidós autores contestan a estas preguntas e introducen al lector en el estudio de las redes sociales y el análisis de la mismas desde la diversidad y amplitud en la que es posible aplicar el método, en diversos campos como la economía, sociología, ecología, biología, desarrollo sostenible, salud, comunicación, o educación.

En el primer capítulo se introduce al lector directamente en el ARS, comenzando con la definición del concepto de red social y su evolución histórica, describiendo los conceptos fundamentales del método, hasta llegar a la representación visual de toda la estructura y las relaciones que se establecen, mediante el grafo total. Los autores ponen especial énfasis en describir la centralidad, pues es un componente esencial que determina la posición del individuo en la red. El capítulo cierra con los tipos de redes que se pueden dar en función de la naturaleza de los actores, la perspectiva del estudio, el tipo de relaciones y los subgrupos y subestructuras. Tras esta introducción, se muestra el amplio campo de aplicación del método ARS en distintos ámbitos.

El primer ámbito en el que se describe el método ARS es el del capital social, dado la enorme vinculación de este concepto con las redes relacionales. Según Florín y otros (2003) el capital social es la red de relaciones de una organización y sus individuos, la cual le proporciona valor al permitirle el acceso y la movilización de recursos. Estas relaciones pueden ser beneficiosos tanto para un colectivo o grupo de empresas, como para los actores individuales de la red (Lin, 2001). Los autores, usando el ARS, analizan el capital social desde una perspectiva organizacional, describiendo cómo son las relaciones individuales y colectivas, y el impacto de estas en crear ventajas competitivas tanto para los individuos como para las organizaciones. Inicialmente el 
análisis se realiza por separado, desde una perspectiva macro para el capital social colectivo, y una perspectiva micro para el capital social individual o diferencial, proponiendo finalmente un modelo que integra ambas dimensiones y que es usado para explicar las ventajas competitivas de las organizaciones.

La aplicación del ARS a los Sistemas Socio-Ecológicos (SSE), aunque reciente, ha supuesto una gran aportación teórica y metodológica. Los autores se centran en este capítulo en el ámbito de la gobernanza de los SSE, y la explicación de las técnicas y métodos aplicados en este ámbito. La recogida de información que proporciona el ARS desde los niveles globales e individual de los SSE permite identificar, entre otros, los actores con mejores recursos, los mejor posicionados y los que pueden impulsar o bloquear acciones colectivas para la buena gobernanza de los SSE. Se muestran los grandes avances obtenidos de las investigaciones de SSE con ARS, los desafíos para la comunidad científica que trabaja en redes en este ámbito, y la necesidad de renovar las teorías existentes, apostando por nuevos desarrollos, que mejorarían el conocimiento y la gobernanza de estos sistemas.

La cooperación interempresarial en el ARS es enmarcada en el concepto más amplio de las relaciones interorganizativas, introduciendo al lector en el concepto de cooperación, sus tipos y las formas más habituales que presenta. Se analizan los tipos de redes empresariales, comparando el análisis tradicional de la cooperación entre empresas y las relaciones interorganizativas en el nuevo modelo relacional, el de "las redes estratégicas", frente al modelo atomista anterior. Puesto que de la participación de una empresa en redes organizativas nacen los recursos de red (Gulati, 1999), y estos determinan el éxito empresarial, es necesario estudiar como las empresas pueden acceder y movilizar estos recursos de red a través de sus relaciones de cooperación y aprovechamiento de los mismos. El ARS es un método especialmente adecuado, que permiten el análisis de datos relacionales, contribuye al estudio de los recursos de red y a explicar convenientemente la cooperación interempresarial.

Con un enfoque más aplicado, en el libro se describen tres casos prácticos de análisis de redes organizativas, en los que se trata de comprender el comportamiento de las organizaciones en un sector de actividad y un contexto específico. Los casos que se incluyen son: intervención con personas inmigrantes, intervención familiar y la industria cultural en el sector de la danza, presentando las conclusiones obtenidas para cada uno de ellos. Los resultados obtenidos muestran entre otros, como se aglutinan las relaciones, y que parte de ellas ocupan un lugar de centralidad, intermediación o periferia dentro de la red, la vinculación entre las entidades en 
función de si es un ámbito público o privado, y las consecuencias derivadas de las dinámicas de intercambio que se producen entre agentes y organizaciones sociales y culturales en el ámbito comunitario, como es el acceso a mayor información y recursos para el desarrollo de la organización.

Los medios sociales en internet son presentados en el libro como los nuevos modelos de comunicación multidireccional que convierten a los individuos en micromedios, frente a la comunicación unidireccional de los medios profesionales, en los que somos relegados a audiencias receptivas, aunque no siempre ni por completo pasivas (Thompson, 1999). En este capítulo, se analiza la estructura de las relaciones en redes en Twitter, como red social online más destacada y dinámica del momento, y como, mediante el ARS es posible visualizar este "lugar sin espacio", el ciberespacio. El autor, incluye varias figuras que sirven de ejemplo para demostrar el potencia visual de ARS, aplicado al caso de la red de una marca comercial y la red de una comunidad temática. Se cierra el capítulo destacando, que a pesar de que el ARS proporciona una forma de medir con precisión la cantidad de relaciones que se dan en la red, no aporta una explicación cualitativa de los intercambios que se producen, por lo que nuevas investigaciones y metodologías, como la "netnografía" (Del-Fresno, 2011) son necesarias y complementarias al ARS.

Como contribución al desarrollo territorial sostenible, se describe en el capítulo siete la Plataforma Audiovisual sobre Tecnología IP (AVIP). Esta plataforma ha sido desarrollada por la UNED, está sustentada en la "presencialidad virtual" , y puede definirse como una red nacional de colaboración, en la que se ofrecen servicios de formación, información y comunicación para la comunidad universitaria. Se detalla la caracterización de la plataforma, los objetivos que se pretendían cumplir, y se realiza un análisis de la metodología aplicada para alcanzarlos. En el análisis de los resultados obtenidos a través de diversas herramientas, entre las que cabe destacar el método de ARS a través de Ghepi, se muestra como AVIP, mediante el acceso a los contenidos disponibles en los repositorios digitales y el trabajo colaborativo en red, contribuye al desarrollo territorial sostenible, entre otros, al fomentar la igualdad de oportunidades y ganancia en aspectos económicos, como reducción de desplazamientos y mejora del medio ambiente.

Para comprender las redes de relación en el ámbito científico los autores se plantean contestar a una serie de interrogantes sobre la colaboración científica. Se revisan previamente los conceptos esenciales de la colaboración científica desde una perspectiva de red, centrándose en la coautoría, como medida más normalizada y frecuente en este contexto. Desde la coautoría los autores establecen los tipos de 
redes, cuáles son sus características, y que información se integra dentro de las mismas. El último apartado del capítulo describe la aplicación del ARS en las redes de coautoría, describiendo el tipo de redes y la calificación de la colaboración, los modos de las matrices existentes, y que información se integra en la red para un mayor conocimiento.

Los procesos de transmisión de la información, la creación y transferencia del conocimiento juegan un papel determinante en nuestra sociedad actual, y tienen efectos importantes en el comportamiento de los individuos. Se analiza como a través del ARS se puede determinar como los procesos de la transmisión de información y de comunicación en una determinada red, tienen una repercusión importante en la estructura social. Estos procesos son estudiados con ejemplos de redes de información existentes, y centrándose en la que actualmente se considera la más relevante, la World Wide Web.

Las posibilidades que ofrece el ARS en la disciplina de la epidemiología son analizadas en función de la importancia de variables y factores de carácter social, y su influencia sobre la salud. Para ello se ofrece una panorámica general de los diferentes estudios y teorías que han puesto de manifiesto la relación entre lo biológico y lo social. El ARS permite cuantificar y evaluar, entre otras, las vías de transmisión potenciales que existen entre los individuos de una comunidad, permitiendo la comprensión de la distribución de la infección en un contexto específico, y el conocimiento de las variables reticulares, contextuales y sociales que favorecen el contagio. Este conocimiento permite el establecimiento de modelos de predicción de la propagación de las epidemias, y a través de que redes sociales se producen las conductas de riesgo.

En el área educativa desde la perspectiva de redes sociales los autores se centran en el cambio educativo, y cómo es necesario tener en cuenta la interacción de todos los actores mediante las relaciones interpersonales que se establecen, que pueden moderar, influir y determinar la velocidad y profundidad de dicho cambio. La perspectiva que se aborda para comprender las mejoras educativas y su consecuente transformación, es entender que estos sistemas están interconectados y son interdependientes. Remarcar la importancia a la hora de analizar el funcionamiento del cambio educativo, de la existencia de dos tipos de sistemas que se dan en estas estructuras organizativas, los formales e informales, y como la teoría de redes aporta una mayor comprensión de los mismos. Se cierra el capítulo aportando una serie de líneas de investigación futura, que exploren los efectos de la estructura global en temas relevantes dentro de la educación como campo de estudio. 
Esta obra refleja fielmente su titulo "Conectados por redes sociales" e ilustra el potencial y posibilidades que tiene el método de Análisis de Redes Sociales en cualquier campo de aplicación, uniendo en una sola obra a veinticinco investigadores de diversas disciplinas. El carácter didáctico del libro, con casos prácticos y ejemplos, y de fácil lectura y comprensión se posiciona como guía para profesionales y estudiantes de diferentes disciplinas, y para todos aquellos que desconocen la increíble potencialidad de esta metodología de investigación. La actual revolución tecnológica está cambiando nuestro entorno laboral, social, cultural y profesional, y el impacto en nuestra vida profesional promete ser mucho más profundo. Las redes sociales online pronto construirán un nuevo entorno laboral, donde la conectividad será equivalente a eficiencia profesional, configurando nuestra forma de entender el trabajo. Las redes sociales on-line son una fuente más de información cada vez mas relevante, que complementa con las que actualmente se superponen a las que llegan del entorno tradicional. La clave está ahora en las redes online, como entorno de evolución y reinvención profesional. Las nuevas generaciones de estudiantes cuentan ya con un manual introductorio que les muestra el abanico de posibilidades que ofrece Análisis de Redes Sociales.

\section{Referencias bibliográficas}

Castells, M. (1997). La era de la información. Economía, Sociedad y Cultura. Vol. 3. Fin de milenio. Madrid: Alianza editorial.

Catells, M. (2009). Comunicación y poder. Madrid: Alianza Editorial.

Del-Fresno, M. (2011). Netnografía. Barcelona: UOC.

Florin, J., Lubatkin, M., \& Schulze, W. (2003). A social capital model of high growth ventures. Academy of Management Journal, 3(46), 374-384.

Gulati, R. (1999). Network location and learning: the influence of network resources and firm capabilities on alliance formation. Strategic Management Journal, 20, 397-420.

Lin, N. (2001). Building a network theory of social capital. En: N. Lin; K. Cook \& R. S. Burt (eds.). Social capital: theory and research. Aldine de Gruyter.

Thompson, J. (1999). Los media y la modernidad. Barcelona: Paidós. 